\title{
JOSEFINA RAMOS DE COX
}

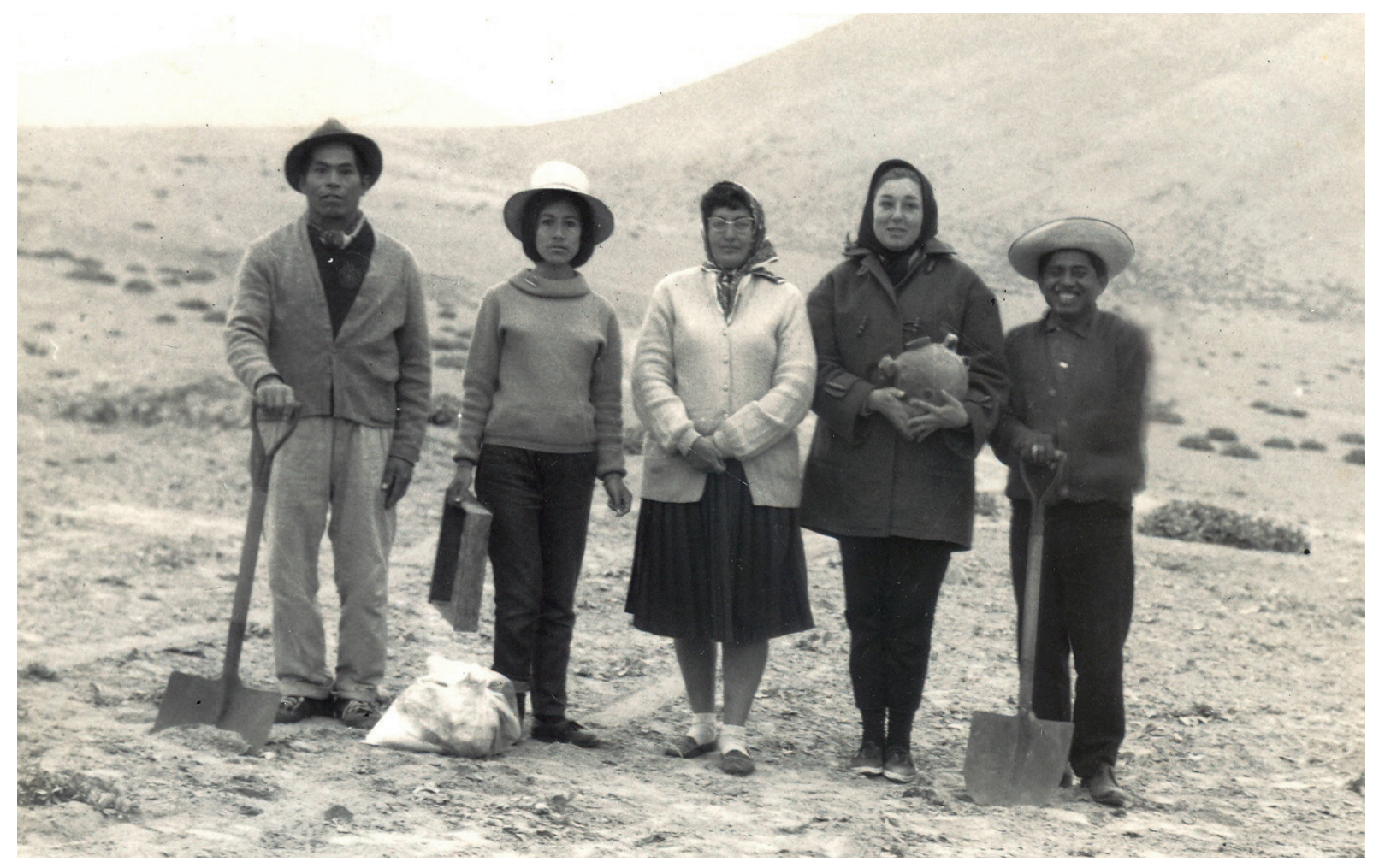

Fotografía de inicios de la década del setenta. A la izquierda don Eugenio Huzco (asistente de campo para el cuidado de las huacas y apoyo en las excavaciones), doctora Hilda Vidal, antropóloga física junto a la doctora Josefina Ramos. A la derecha figuran doctora arqueóloga Gabriela Shoerwel y su chofer.

Fuente: Archivo institucional del Museo de Arqueología Josefina Ramos de Cox-IRA-PUCP, 2016.

Notable maestra e investigadora, su labor académica se caracterizó por una permanente reflexión sobre la valoración del hombre peruano y su cultura. Piura, su ciudad natal, fue el primer escenario para esta búsqueda del devenir histórico de nuestros antepasados. En su tesis de bachiller del año de 1950 cita lo siguiente: "Nuestros antepasados no silenciaron su conocimiento: el español en líneas, la fue descubriendo, i el indio la continuó viviendo. Como descendientes de este mestizaje nos toca reunir a las dos herencias en una, interpretando esa síntesis cultural" (julio, 1950).

Como estudiante (1946-1952) en la Universidad Nacional Mayor de San Marcos y en la Pontificia Universidad Católica del Perú, se graduó de abogada y de doctora en Letras con mención en Historia respectivamente. En este proceso de formación y ejercicio académico, entre los años 50 y 70, fue construyendo una ruta significativa como peruanista: en 1947 ingresó a la Unión Nacional de Estudiantes Católicos (UNEC) e integró la cédula de Derecho, y se constituyó como presidenta del consejo local en Lima. En adelante el camino hacia el servicio del Perú se hace intenso e infatigable, porque inicia su tarea interdisciplinaria e interinstitucional, sello que la caracterizó a lo largo de su trayectoria académica. Así, participó como miembro activo en diferentes congresos nacionales e internacionales, entre ellos el Congreso Internacional de Peruanistas; la Mesa redonda de Ciencias: Prehistoria y Arqueología, en la UNMSM; la Primera Jornada Arqueológica, del Instituto Riva Agüero; y el Congreso de Arqueología en Río Negro, Argentina.

Su mirada como jurista y educadora la llevó a comprometerse con funciones de carácter público; formó parte del Patronato Nacional de Arqueología como miembro del Consejo Técnico de Arqueología en el Instituto Na- 
cional de Arqueología. Las evaluaciones de proyectos extranjeros y nacionales nos descubren una maestra en permanente actualización con las corrientes teóricas de la época y la legislación patrimonial. Cabe destacar su actuación calificadora de los proyectos de la Misión Junius Bird, la Misión Ford, los estudios de Patterson en la costa Central, Jhon Rowe en el Cusco y Dorothy Menzel en la costa sur.

Su experiencia en la docencia escolar culminó con la gestión y fundación del Colegio Cooperativo Arco Iris, proyecto de importancia social y pedagógica, un ejemplo para iniciar con los niños y jóvenes la idea de comunidad y cooperación en el marco del diálogo intercultural.

\section{Pensar el Perú y Lima desde la Arqueología: creación del Seminario de Arqueología y el museo del IRA-PUCP}

En el Instituto Riva Agüero de la Pontificia Universidad Católica del Perú, fundó el Seminario de Arqueología en el año 1956, y en 1970 el Museo de Arqueología. A través de estas instituciones desarrolló una intensa actividad arqueológica en la cual la investigación fue a la par con la idea de la defensa y preservación del patrimonio arqueológico, un enfoque sin duda precursor del reto que hoy vive la disciplina arqueológica para la preservación de los sitios y monumentos, especialmente aquellos ubicados en Lima.

En el Seminario de Arqueología impulsó la formación de equipos interdisciplinarios y multidisciplinarios entre estudiantes y profesionales que debían aportar desde sus disciplinas ideas para resolver los problemas que demandaban entender el Perú a partir de las fuentes arqueológicas. Para lograr este fin inicialmente creó grupos de estudio, y talleres, y contribuyó a fortalecer la escuela internacional de temporada mediante un convenio con Fullbrigh especialmente dirigido a estudios peruanistas. El seminario se convirtió en un foro permanente en el que antropólogos, historiadores, biólogos, ingenieros, arquitectos y filósofos debatían, inspirados en la Arqueología la temática del Perú. Los grupos de estudio que más recuerdo fueron el Seminario de Símbolos, de Patrones de Asentamiento, de Antropología Física, de Etnohistoria, de Legislación Arqueológica, de Arqueología Bíblica, etc. El debate metodológico sobre el registro, inventario y catalogación era permanente y dirigido a la salvaguarda de la colección del seminario procedente de las investigaciones de campo realizadas en Maranga y Tablada de Lurín. Priorizó prospecciones y excavaciones de campo en los valles del Chillón, Rímac y Lurín, que facilitaron la planificación y ejecución del catastro arqueológico del Rímac.

La lectura transversal del Perú y los resultados de las investigaciones en Lima motivaron a la doctora a establecer nuevas áreas de estudio, desarollar proyectos nacionales e internacionales. La costa en su amplitud, las cuencas serranas de Lima, la ruta Ica-Cusco y la cuenca del río Tambopata en la selva sur-Madre de Dios surgen como escenarios que tienen el objetivo de dar respuestas a las interrogantes sobre las interrelaciones ancestrales entre los pueblos de la costa, de la sierra de la selva. Con el auspicio de la fundación VW se llevaron a cabo investigaciones arqueológicas en la costa norte y central del Perú. En la costa sur se realizó el proyecto Huayurí-Ica mediante el convenio Peruano-Argentino, que permitió ampliar los estudios antes realizados en la zona de Mala y León Dormido. En la Selva sur, mediante convenio con el Instituto de Estudios Tropicales, se llevó a cabo excavaciones arqueológicas en el Lago Sandoval, en el departamento de Madre de Dios.

Para divulgar las investigaciones arqueológicas de los miembros del seminario, creó el Boletín de Arqueología, donde escribió sobre la interdisciplinariedad entre la Arqueología, la Astronomía, la Etnohistoria, la Ecología, etc. Cabe resaltar la divulgación de los primeros estudios del aporte de la informática aplicada al registro, inventario y catalogación de la cerámica estudiada en el valle del Rímac/Huacas Pando.

En el museo inició una permanente comunicación educativa con el público, a través de la exposición de testimonios arqueológicos identitarios de Lima en su devenir local y regional, y el impacto de lo. Finalmente, dejó una reflexión patrimonialista: "la huaca Milenaria es el testimonio inicial de una realidad que marcha. Triste sería que por incuria o ignorancia el Perú se quedara colgado en el tiempo, inseguro y roto" (1964, Archivo Josefina Ramos de Cox).

Dra. Inés Del Águila Ríos 